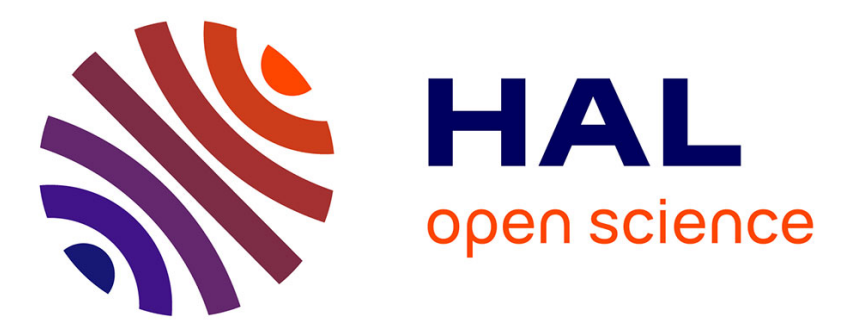

\title{
Experimental photoacoustic observation of the photogenerated excess carrier influence on the thermoelastic response of n-type silicon
}

D D Markushev, S M Aleksić, D S Pantić, S P Galović, D M Todorović, Jose Ordonez-Miranda

\section{To cite this version:}

D D Markushev, S M Aleksić, D S Pantić, S P Galović, D M Todorović, et al.. Experimental photoacoustic observation of the photogenerated excess carrier influence on the thermoelastic response of n-type silicon. Journal of Applied Physics, 2020, 128, 10.1063/5.0015657 . hal-03017602

\section{HAL Id: hal-03017602 https://hal.science/hal-03017602}

Submitted on 21 Nov 2020

HAL is a multi-disciplinary open access archive for the deposit and dissemination of scientific research documents, whether they are published or not. The documents may come from teaching and research institutions in France or abroad, or from public or private research centers.
L'archive ouverte pluridisciplinaire $\mathbf{H A L}$, est destinée au dépôt et à la diffusion de documents scientifiques de niveau recherche, publiés ou non, émanant des établissements d'enseignement et de recherche français ou étrangers, des laboratoires publics ou privés. 


\section{Experimental photoacoustic observation of the photogenerated excess carrier influence on the thermoelastic response of n-type silicon}

Cite as: J. Appl. Phys. 128, 095103 (2020); https://doi.org/10.1063/5.0015657

Submitted: 01 June 2020. Accepted: 15 August 2020 . Published Online: 03 September 2020

D. K. Markushev (D), D. D. Markushev, S. M. Aleksić ${ }^{(D)}$, D. S. Pantić ${ }^{(D, ~ S . ~ P . ~ G a l o v i c ́ ~}{ }^{(D)}$ D. M. Todorović, and J. Ordonez-Miranda (iD)

\section{Lock-in Amplifiers up to $600 \mathrm{MHz}$}
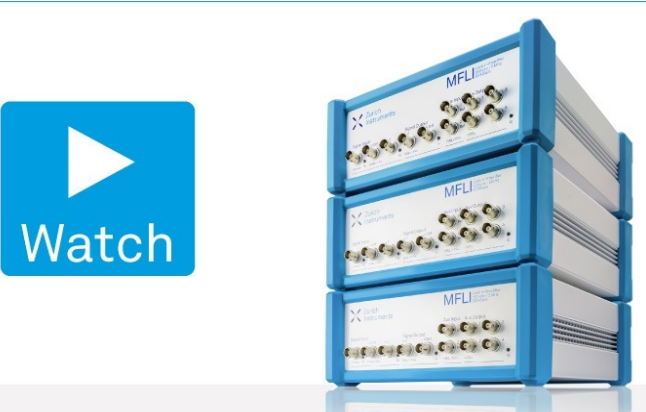


\title{
Experimental photoacoustic observation of the photogenerated excess carrier influence on the thermoelastic response of $n$-type silicon
}

Cite as: J. Appl. Phys. 128, 095103 (2020); doi: 10.1063/5.0015657

Submitted: 1 June 2020 - Accepted: 15 August 2020 .

Published Online: 3 September 2020

D. K. Markushev, ${ }^{1}$ (D) D. D. Markushev, ${ }^{2}$ S. M. Aleksić, ${ }^{1, a)}$ (D) D. S. Pantić, ${ }^{1}$ (D) S. P. Galović, ${ }^{3}$ (D) D. M. Todorović, ${ }^{4}$ and J. Ordonez-Miranda ${ }^{5}$ (D)

\author{
AFFILIATIONS \\ ${ }^{1}$ Faculty of Electronic Engineering, University of Niš, Aleksandra Medvedeva 1418000 , Niš, Serbia \\ ${ }^{2}$ Institute of Physics, National Institute of the Republic of Serbia, University of Belgrade, Pregrevica 118 11080, Belgrade-Zemun, \\ Serbia \\ ${ }^{3}$ Vinča Institute of Nuclear Sciences, P.O. Box 522, 11001 Belgrade, Serbia \\ ${ }^{4}$ Institute for Multidisciplinary Research, University of Belgrade, P.O. Box 33, 11030 Belgrade, Serbia \\ ${ }^{5}$ Institut Pprime, CNRS, Université de Poitiers, ISAE-ENSMA, F-86962 Futuroscope Chasseneuil, France
}

\begin{abstract}
${ }^{a)}$ Author to whom correspondence should be addressed: sanja.aleksic@elfak.ni.ac.rs
ABSTRACT

Based on the experimental and theoretical signals of an open photoacoustic cell operating with modulation frequencies from $20 \mathrm{~Hz}$ to $20 \mathrm{kHz}$, a significant contribution of photogenerated excess carriers on the thermal and thermoelastic responses of an n-type silicon plate is observed for the very first time. This is achieved by comparing the measured amplitude and phase of the photoacoustic signal with their corresponding theoretical thermoelastic counterparts, for high enough modulation frequencies mainly. It is shown that the amplitude of the thermoelastic component of plasma-thin samples varies about two orders of magnitude with respect to the corresponding one of plasma-thick samples. Furthermore, we find a maximal temperature difference $\Delta \mathrm{T}=-35 \mathrm{nK}$ between the illuminated and non-illuminated sample surfaces, which shows that thin silicon plates with excess carriers could be used as heat sinks.
\end{abstract}

Published under license by AIP Publishing. https://doi.org/10.1063/5.0015657

\section{INTRODUCTION}

As is well-known, the light-matter interaction in semiconductors leads to light absorption followed by a non-radiative excitation state resulting in the sample temperature rise. ${ }^{1-3}$ If the energy of a photon is equal to or greater than the bandgap of the illuminated material, the photon excites an electron into the conduction band and generates an electron-hole pair. ${ }^{2,3}$ These pairs are called photogenerated or excess carriers, their density takes its highest value at the illuminated surface, where the majority of light is absorbed, and, therefore, they induce various effects in semiconductors. ${ }^{4-6}$

Photogenerated excess carriers can change the heat transport dynamics and temperature profiles inside semiconductors. ${ }^{7-13}$ In photoacoustics (PA), for instance, some theoretical studies showed that the number of photogenerated excess carriers generated in both $\mathrm{n}$ - and p-type semiconductors illuminated with a modulated light source increases with the light absorption, and they are able to significantly change the temperature distribution at the surface and inside the materials. ${ }^{14-18}$ The experimental observation of these effects still remains a challenge due to the separate analysis of either the amplitude or phase of the PA signal in a relatively short range of modulation frequencies. ${ }^{19-23}$ The simultaneous monitoring of both of these signals in a wide interval of modulation frequencies is thus desirable to observe the effects of photogenerated excess carriers.

In our recent theoretical studies of n-type silicon circular plates, ${ }^{22,23}$ we noticed that the impact of photogenerated excess carriers on their PA response depends mostly on the semiconductor surface quality determined by the surface recombination speed $s$, the sample thickness $l$, and the carrier diffusion length $L_{p}=\sqrt{D_{p} \tau}$, with $D_{p}$ being the diffusion coefficient of the minority carriers with 
lifetime $\tau$. Favorable conditions for the observation of this impact were found when the illuminated sample surface is passivated $\left(s_{1} \rightarrow 0\right)$, while the non-illuminated one remains active $\left(s_{2} \gg 0\right){ }^{22}$ In this latter case, the PA response of plasma-thin semiconductors $\left(l / L_{p}<1\right)$ was found to be completely different than that of plasma-thick ones $\left(l / L_{p}>1\right){ }^{23}$ More importantly, the modulated temperature component $T_{s}(l)$ at the non-illuminated back surface of a plasma-thin sample can be higher than the corresponding one $T_{s}(0)$ at its illuminated front surface, within a wide range of modulation frequencies. ${ }^{23}$ In other words, the excess carriers of plasmathin samples can be used to remove the absorbed light energy from the illuminated surface and transfer it to the non-illuminated one, and, therefore, these materials could become efficient heat sinks. On the other hand, taking into account that the amplitude of the thermoelastic (TE) component of the PA signal of plasma-thin samples is significantly attenuated at low modulation frequencies $(f<1 \mathrm{kHz})$ and exhibits a typical peak-like shape at high ones $(f>1 \mathrm{kHz}),{ }^{22,23}$ the bending of these materials can show up at high modulation frequencies, mainly.

In the present work, the theoretical observations reported in our previous articles ${ }^{22,23}$ for the influence of the excess carrier are experimentally studied by means of an open-cell PA experimental setup operating in the transmission configuration. ${ }^{21-23}$ The amplitude and phase of the PA response of n-type silicon plates of different thicknesses are measured for modulation frequencies ranging from $20 \mathrm{~Hz}$ to $20 \mathrm{kHz}$ and their values are found to be in good agreement with theoretical predictions. For plasma-thin samples, the expected amplitude attenuation of the TE signal is observed along with the dominant thermal diffusion (TD) component of the total PA signal. Based on the analysis of the PA signals at the illuminated and non-illuminated sample surfaces, the fingerprints of the excess carriers on the PA response are confirmed.

\section{EXPERIMENTAL SETUP}

The measurements were carried out with an open-cell experimental setup ${ }^{24-26}$ using three $\mathrm{Si}$ circular plates of radius $R_{\mathrm{s}}=4 \mathrm{~mm}$ and thicknesses $l_{1}=150 \mu \mathrm{m}, l_{2}=85 \mu \mathrm{m}$, and $l_{3}=30 \mu \mathrm{m}$; as samples mounted directly on top of a microphone. All plates were prepared from the same non-degenerated n-type Si wafer $(3-5 \Omega \mathrm{cm},\langle 100\rangle$ oriented) having the electron concentration of $n_{0}=9 \times 10^{20} \mathrm{~m}^{-3}$. ${ }^{29}$ The samples thus obtained were excited with a laser diode emitting light at $656 \mathrm{~nm}$ with an intensity of $I_{0}=10 \mathrm{~W} / \mathrm{m}^{2}$ and photon energy $\varepsilon$ greater than the Si bandgap $\varepsilon_{g}$. The absorption coefficient of our $\mathrm{Si}$ samples at the diode wavelength is $\beta=2.58 \times 10^{5} \mathrm{~m}^{-1}$. The laser beam intensity was modulated with a homemade current modulator in the frequency range of $20 \mathrm{~Hz}-20 \mathrm{kHz}$, which was monitored by a BPW34 photodiode of high speed and accurate radiant sensitivity. The irradiated plate surface was polished, while the back was etched. Data acquisition was performed by using an Intel $82801 \mathrm{Ib} / \mathrm{ir} / \mathrm{ih}$ hd PC audio controller, and the amplitude and phase of the PA signal detected by a Jin In Electronics Co. model ECM30 electret microphone were extracted by means of a software emulating a lock-in amplifier.

A schematic diagram illustrating the main components of our open-cell PA experimental setup is depicted in Fig. 1. The

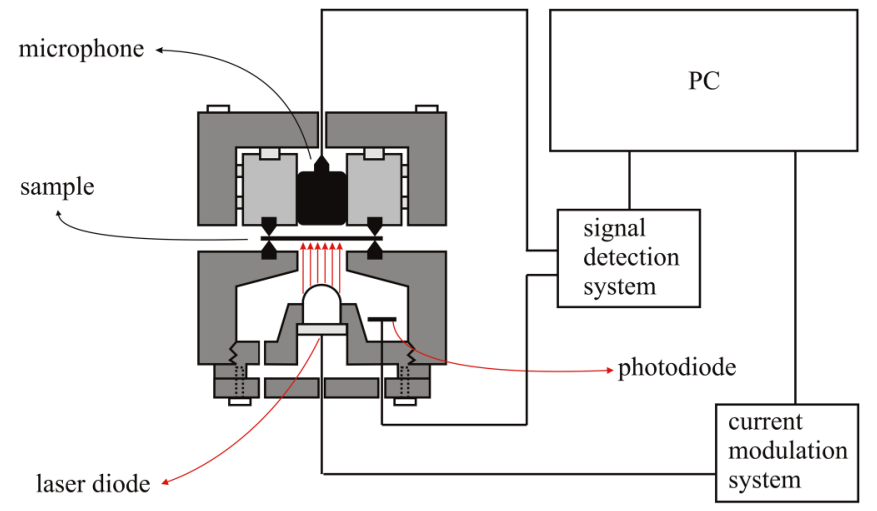

FIG. 1. Scheme of the open photoacoustics cell used in our experiments under the transmission configuration.

transmission configuration was adopted to assure 1D heat conduction along the uniformly irradiated plate, which allows using simple theoretical models to interpret the obtained experimental data. Taking into account that frequency response of the microphone and accompanying electronics has bandpass (electronic filter) characteristics that depend on the design, manufacturing, and diaphragm properties, we applied a standard signal correction procedure ${ }^{27,28}$ to obtain undistorted signals accurately representing the physical characteristics of the considered samples.

\section{SIGNAL CORRECTION PROCEDURE}

The measured open-cell PA response $S_{\exp }(f)$ can be represented in the following form: ${ }^{29}$

$$
S_{\exp }(f)=A_{\exp }(f) \exp \left[i \varphi_{\exp }(f)\right],
$$

where $A_{\exp }(f)$ and $\varphi_{\exp }(f)$ are its respective amplitude and phase, $i$ is the complex unit, and $f$ is the modulation frequency. In an ideal case, $S_{\exp }(f)$ is equal to the "true" PA signal $\delta p_{\text {total }}(f)$ generated by the investigated sample. Unfortunately, noise and signal distortion often mask $\delta p_{\text {total }}(f)$, so their analysis is an unavoidable task in accurate and precise PA measurements. ${ }^{27,28}$

Main types of noise in PA measurements are flicker noise $N_{\mathrm{FN}}(f)$ and coherent signal deviations $N_{\mathrm{CSD}}(f)$. Flicker noise is typically generated by the random and nonreducible microphone noise. Coherent signal deviations, on the other hand, represent unwanted coherent disturbances originated by the LED current modulation system and they can, fortunately, be avoided or subtracted. The main types of PA signal distortions are originated by the filtering of instruments used in measurements, usually microphone and PC sound card (lock-in emulator). At relatively low modulation frequencies, electronic filtering dominates as a main source of distortion due to the instrumental $R C$ characteristics. These filtering effects can be accounted for using a first-order highpass filter described by a cascade system transfer function, which 
can be written as follows: $:^{27,28}$

$$
H_{e}(f)=-\frac{f}{\left(f_{1}+i f\right)} \cdot \frac{f}{\left(f_{2}+i f\right)},
$$

where $f_{1}$ and $f_{2}$ are the characteristic frequencies of the microphone and sound-card, respectively. At relatively high frequencies, on the other hand, acoustic filtering becomes the main source of distortion. Usually, one or two peaks emerge due to the acoustic resonances closely connected to the microphone geometry and microphone membrane features. Taking into account that the transfer functions of physically different systems can be identical, the acoustic properties of the microphone can be represented by the following series of second-order low-pass filters: ${ }^{28}$

$$
H_{a}(f)=\frac{f_{3}^{2}}{f_{3}^{2}+f_{3} \delta_{3} i f-f^{2}}+\frac{f_{4}^{2}}{f_{4}^{2}+f_{4} \delta_{4} i f-f^{2}},
$$

where $f_{3}$ and $f_{4}$ are the characteristic frequencies of the first and second peaks of the microphone, while $\delta_{3}$ and $\delta_{4}$ are their corresponding dumping factors.

Taking into account the main types of noise and signal distortions, it is clear that the correction of $S_{\exp }(f)$ is needed to obtain $\delta p_{\text {total }}(f)$. If we measure and subtract $N_{\mathrm{CSD}}(f)$, assuming that $N_{\mathrm{FN}}(f)$ is negligible, the values of $S_{\exp }(f)$ can be fitted with $S_{\text {fitt }}(f)$ given by $^{27,28}$

$$
S_{\text {fitt }}(f)=\delta P(f) \cdot H(f),
$$

where $H(f)=H_{e}(f) \cdot H_{a}(f)$ is the instrumental disturbance and $\delta P(f)$ is the assumed undistorted signal $\left(\delta P(f) \approx \delta p_{\text {total }}(f)\right)$. In general, the frequency dependence of $\delta P(f)$ can be modeled as the power law $\delta P(f) \sim f^{n}$, where $n$ is a given exponent equal to $n_{A}$ for the amplitude and $n_{\varphi}$ for the phase. These latter two parameters can be found by linearizing the expressions for $A_{\exp }(f)$ and $\varphi_{\exp }(f)$ in Eq. (1).

To find the sample properties, one has to fit $\delta P(f)$ to the $\delta p_{\text {total }}(f)$ function determined by the well-known composite piston theoretical model, which, for semiconductors as the n-type Si considered in this work, establishes that ${ }^{19-21,27-29}$

$$
\delta p_{\text {total }}(f)=\delta p_{\mathrm{TD}}(f)+\delta p_{\mathrm{TE}}(f)+\delta p_{\mathrm{PE}}(f)
$$

where

$$
\begin{gathered}
\delta p_{\mathrm{TD}}(f)=\frac{\gamma_{\mathrm{g}} p_{0} \sqrt{D_{g}}}{l_{c} T_{0} \sqrt{2 \pi i f}} T_{s}(l), \\
\delta p_{\mathrm{TE}}(f)=\alpha_{T} \frac{\gamma p_{0}}{V_{0}} \frac{3 \pi R_{s}^{4}}{l^{3}} \int_{0}^{l}\left(x-\frac{l}{2}\right) T_{s}(x) \mathrm{d} x,
\end{gathered}
$$

and

$$
\delta p_{\mathrm{PE}}(f)=d_{n} \frac{\gamma p_{0}}{V_{0}} \frac{3 \pi R_{s}^{4}}{l^{3}} \int_{0}^{l}\left(x-\frac{l}{2}\right) \delta n_{p}(x) \mathrm{d} x,
$$

are the thermal-diffusion $\delta p_{\mathrm{TD}}(f)$, thermoelastic $\delta p_{\mathrm{TE}}(f)$, and plasmaelastic $\delta p_{\mathrm{PE}}(f)$ components of the PA signal. Here, $\gamma$ is an adiabatic ratio, $V_{0}$ is the photoacoustic cell volume, $p_{0}$ is the ambient pressure, $T_{s}(x)$ is the dynamic component of the temperature distribution across the sample thickness due to its periodical heating (see the Appendix), $T_{s}(l)$ is the dynamic temperature component at the back sample surface, and $\delta n_{p}(x)$ is the dynamic component of the photogenerated minority carrier density across the sample. According to Eqs. (5)-(8), the adjustment of $\delta P(f)$ to $\delta p_{\text {total }}(f)$ can lead to the determination of the sample properties, such as the thermal diffusivity $D_{\mathrm{T}}$, the excess carrier bulk lifetime $\tau$, the coefficient of thermal expansion $\alpha_{T}$, the surface recombination speeds at illuminated $\left(s_{1}\right)$ and nonilluminated $\left(s_{2}\right)$ surfaces, the coefficient of electronic deformation $d_{n}$, the diffusion coefficient of minority carriers $D_{p}$, etc.

\section{RESULTS AND DISCUSSION}

The measured values of $A_{\exp }(f)$ and $\varphi_{\exp }(f)$ are shown in Figs. 2(a)-2(f) for three samples with thicknesses $l_{1}, l_{2}$, and $l_{3}$. In general, $S_{\exp }(f)$ (black asterisks) accounts for the combination of the undistorted PA signal $\left[\delta P_{\text {total }}(f)\right]$ of the sample (red line) and the instrumental disturbances represented by $H(f)$ (black arrows). ${ }^{27,28}$ Note that the amplitude curves exhibit pretty much the same shape for all thicknesses and modulation frequencies, while the phases do differentiate among them at high enough frequencies. Thinner samples exhibit a higher peak on the phase.

According to Fig. 2 and our experience, $H(f)$ is the main cause of the $\delta p_{\text {total }}(f)$ distortions. The filtering influences of both $H_{e}(f)$ and $H_{a}(f)$ on the amplitude and phase of $S_{\exp }(f)$ are indicated in Figs. 2(a) $-2(\mathrm{f})$ with black arrows.

The $\ln S_{\exp }(f)$ values (Fig. 2-asterisk) are linearized with $S_{\text {fitt }}(f)$ (Fig. 2-blue dashed line) for all thicknesses to $n_{A}=-1.5$, for amplitudes $A(f)$, and $n_{\varphi}=-0.5$, for phases $\varphi(f)$. Simultaneously, deviation parameters are calculated with $H_{e}(f)$ and $H_{a}(f)$ and are presented in Table I.

Rectifying $S_{\text {fitt }}(f)$ by removing all detected $H_{e}(f)$ and $H_{a}(f)$ distortions, an undistorted signal $\delta P(f)$ is obtained (Fig. 2-red line) as an accurate representation of the "true" $\delta p_{\text {total }}(f)$ signal, whose amplitude and phase generated by the investigated Si circular plates are analyzed.

Adjusting $\delta p_{\text {total }}(f)$ to $\delta P(f)$ by using a composite piston theoretical model [Eqs. (5)-(8)] leads to the sample parameter evaluation presented in Table II. ${ }^{29}$ They are the same for all three samples, due to the fact that they are made up of the same wafer. ${ }^{29}$

Based on these results, it is interesting to notice that the calculated value of excess carrier diffusion length is $L_{p}=\sqrt{D_{p} \tau} \approx 85 \mu \mathrm{m}$. It means that the Si sample thickness of $l_{2-}$ $=85 \mu \mathrm{m}$, used previously as the randomly chosen sample, ${ }^{27}$ can be considered here as the boundary thickness between plasma-thick $\left(l_{1}=150 \mu \mathrm{m}\right)$ and plasma-thin $\left(l_{3}=30 \mu \mathrm{m}\right)$ samples. $^{23}$ 

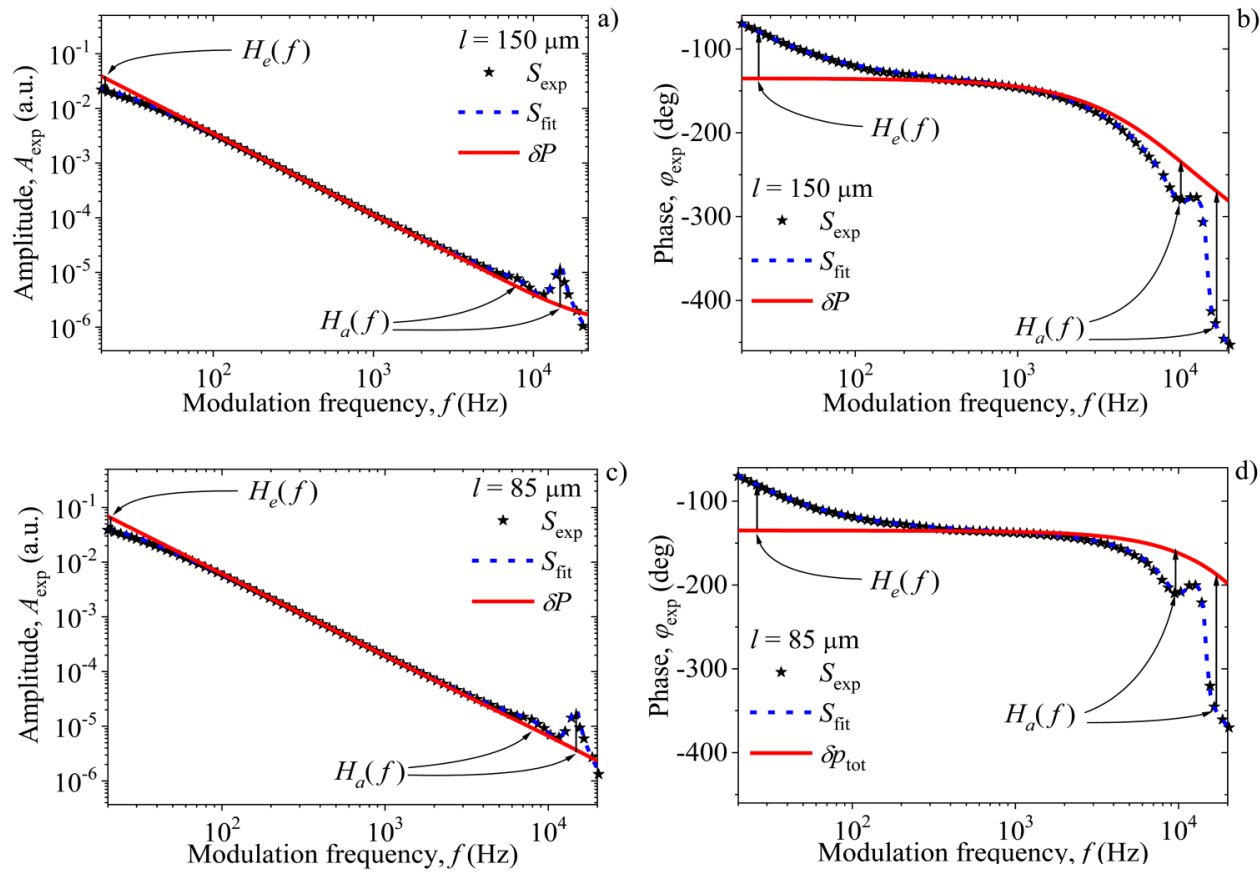

FIG. 2. (a), (c), and (e) Amplitudes $A_{\text {exp }}(f)$ and $(b-f)$ phases $\varphi_{\text {exp }}(f)$ of the measured $S_{\text {exp }}$, fitted $S_{\text {fitt, }}$ and assumed undistorted PA signals $\delta P$ as the functions of modulation frequency $f$. The scales for $A_{\exp }(f)$ and $\varphi_{\exp }(f)$ are the same for all thicknesses $I$ to emphasize their differences.
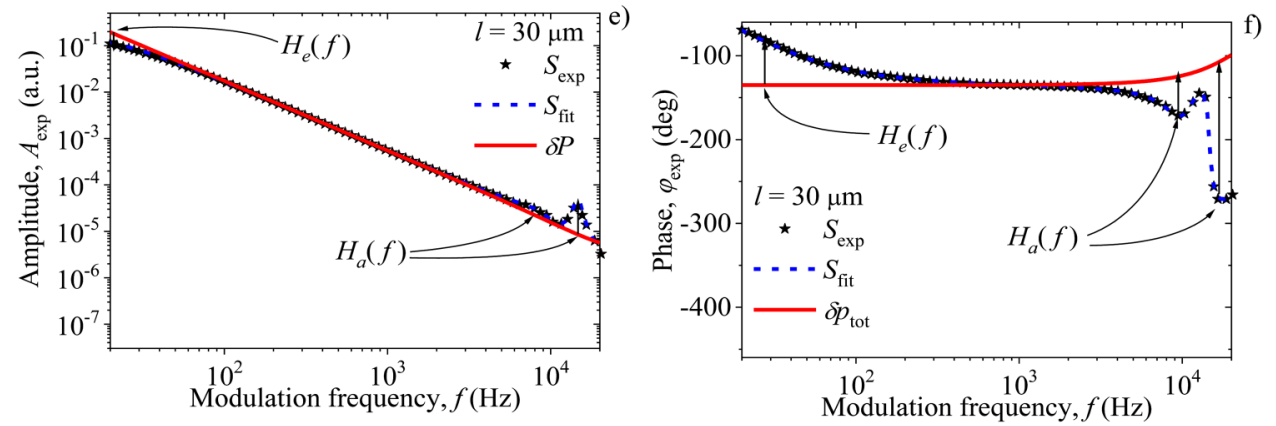

TABLE II. Parameters of silicon samples. ${ }^{29}$

TABLE I. Measuring system characteristic parameters.

\begin{tabular}{cc}
\hline \hline Parameter $\quad \begin{array}{c}\text { Microphone characteristic } \\
\text { frequency, } f_{1}(\mathrm{~Hz})\end{array}$ & Value \\
\hline $\begin{array}{c}\text { PC sound card } \\
\text { characteristic frequency, } f_{2}(\mathrm{~Hz})\end{array}$ & $15 \pm 1$ \\
$\begin{array}{c}\text { Microphone characteristic, first peak frequency, } \\
f_{3}(\mathrm{kHz})\end{array}$ & $9.4 \pm 0.1$ \\
$\quad \begin{array}{c}\text { microphone characteristic } \\
\text { Second peak (cut-off) frequency, } f_{4}(\mathrm{kHz}) \\
\text { First peak damping factor, } \delta_{3}\end{array}$ & $14.7 \pm 0.2$ \\
Second peak damping factor, $\delta_{4}$ & $0.60 \pm 0.05 \pm 0.01$ \\
\hline \hline
\end{tabular}

\begin{tabular}{|c|c|}
\hline Parameter & Value \\
\hline Thermal diffusivity, $D_{T}\left(\mathrm{~m}^{2} \mathrm{~s}^{-1}\right)$ & $(9.0 \pm 0.1) \times 10^{-5}$ \\
\hline Excess carrier bulk lifetime, $\tau$ (s) & $(6.0 \pm 0.2) \times 10^{-6}$ \\
\hline $\begin{array}{l}\text { Coefficient of thermal expansion, } \\
\qquad \alpha_{T}\left(\mathrm{~K}^{-1}\right)\end{array}$ & $(2.6 \pm 0.2) \times 10^{-6}$ \\
\hline $\begin{array}{l}\text { Surface recombination speed at } \\
\text { illuminated front, } s_{1}\left(\mathrm{~m} \mathrm{~s}^{-1}\right)\end{array}$ & $(2.6 \pm 0.2)$ \\
\hline $\begin{array}{l}\text { Surface recombination speed at } \\
\text { non-illuminated back, } s_{2}\left(\mathrm{~m} \mathrm{~s}^{-1}\right)\end{array}$ & $(24 \pm 3)$ \\
\hline $\begin{array}{l}\text { Diffusion coefficient of minority } \\
\text { carriers, } D_{p}\left(\mathrm{~m}^{2} \mathrm{~s}^{-1}\right)\end{array}$ & $(1.2 \pm 0.1) \times 10^{-3}$ \\
\hline $\begin{array}{l}\text { Coefficient of electronic deformation, } \\
\qquad d_{n}\left(\mathrm{~m}^{3}\right)\end{array}$ & $(-9.0 \pm 0.2) \times 10^{-31}$ \\
\hline
\end{tabular}


Now the (a) amplitudes $A(f)$ and (b) phases $\varphi(f)$ and all of its $\delta p_{\mathrm{TD}}(f), \delta p_{\mathrm{TE}}(f)$, and $\delta p_{\mathrm{PE}}(f)$ components are defined [Eqs. (5)-(8)] and depicted in Figs. 3(a)-3(f).

Analyzing Figs. 3(a)-3(f), one can conclude that typical patterns of PA response curves (amplitudes and phases) exist, which can be used to recognize the sample thickness. The $\delta p_{\text {total }}(f)$ amplitude curves look almost the same for all thicknesses, so the analysis of PA components must be taken into the count. In the case of plasma-thick samples, amplitudes of $\delta p_{\mathrm{TD}}(f)$ and $\delta p_{\mathrm{TE}}(f)$ components have an intersection such that $\delta p_{\mathrm{TD}}(f)$ dominates at lower and $\delta p_{\mathrm{TE}}(f)$ dominates at higher frequencies. Intersection does not exist in plasma-thin samples; $\delta p_{\mathrm{TD}}(f)$ dominates at all frequencies. As we predicted in our previous article, ${ }^{22,23}$ the influence of excess carriers as heat carriers can be clearly seen as a strong attenuation of the $\delta p_{\mathrm{TE}}(f)$ amplitude in plasma-thin samples at lower frequencies $(<1 \mathrm{kHz})$ followed by its amplification, e.g., the formation of the peak-like structure (peak beginning in our case) at higher frequencies $(>1 \mathrm{kHz})$ [Figs. 3(a)-3(e)]. In other words, the ratio of thick $\delta p_{\mathrm{TE}}^{150}(f)$ and thin $\delta p_{\mathrm{TE}}^{30}(f)$ thermoelastic amplitudes is almost two orders of magnitude $\left(\delta p_{\mathrm{TE}}^{150}(f) / \delta p_{\mathrm{TE}}^{30}(f) \approx 10^{2}\right)$, much larger than in the "no carriers" escenario $\left(\delta p_{\mathrm{TE}}^{150}(f) / \delta p_{\mathrm{TE}}^{30}(f) \approx 1.2\right){ }^{22,23}$ This fact indicates that the investigated silicon plate, under given experimental conditions, loses its flexibility. At the same time, the $\delta p_{\text {total }}(f)$ phase curves follow some patterns by changing the slopes at higher frequencies [Figs. 3(b)-3(f)]; from negative (plasmathick) to positive (plasma-thin samples). This is a direct influence of $\delta p_{\mathrm{PE}}(f)$ on $\delta p_{\text {total }}(f)$ at higher frequencies, detected for the first time in our experiments. This is the only case where we have a direct influence of excess carriers as charge not heat carriers on our PA response.

It is interesting to take notice that the $\delta p_{\mathrm{PE}}(f)$ component does not change significantly when a sample thickness change, either in amplitude or in phase. The $\delta p_{\mathrm{TD}}(f)$ component changes slightly only in the case of plasma-thick samples, at higher
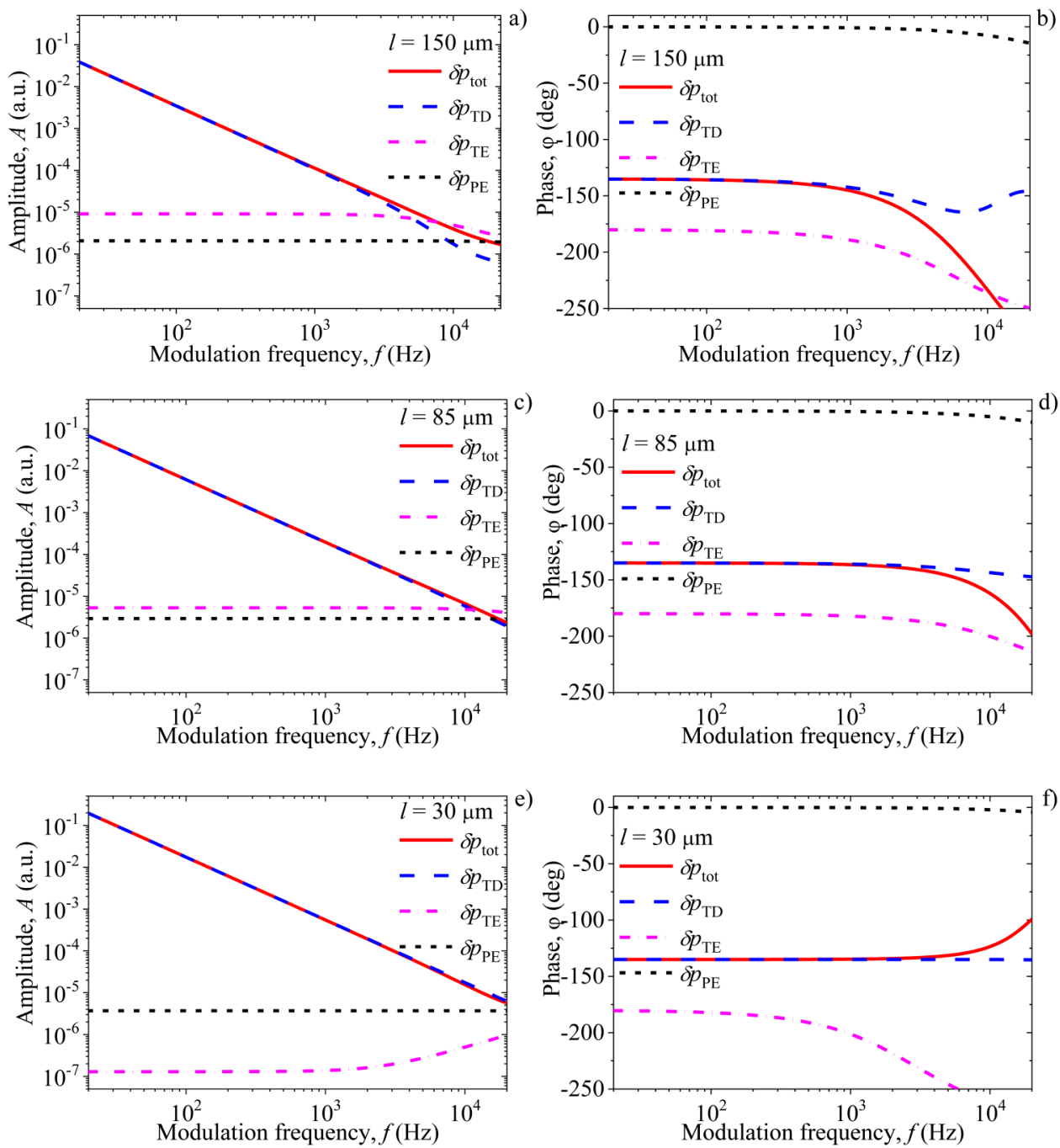

FIG. 3. The results of (a), (c), (e) amplitudes $A(f)$ and $(b),(d)$, and (f) phases $\varphi(f)$ of $\delta p_{\text {total }}$ and its components $\delta p_{\mathrm{TD}}, \delta p_{\mathrm{TE}}$, and $\delta p_{\mathrm{PE}}$ as a function of modulation frequencies $f$, in the case of different sample thicknesses $I$. 

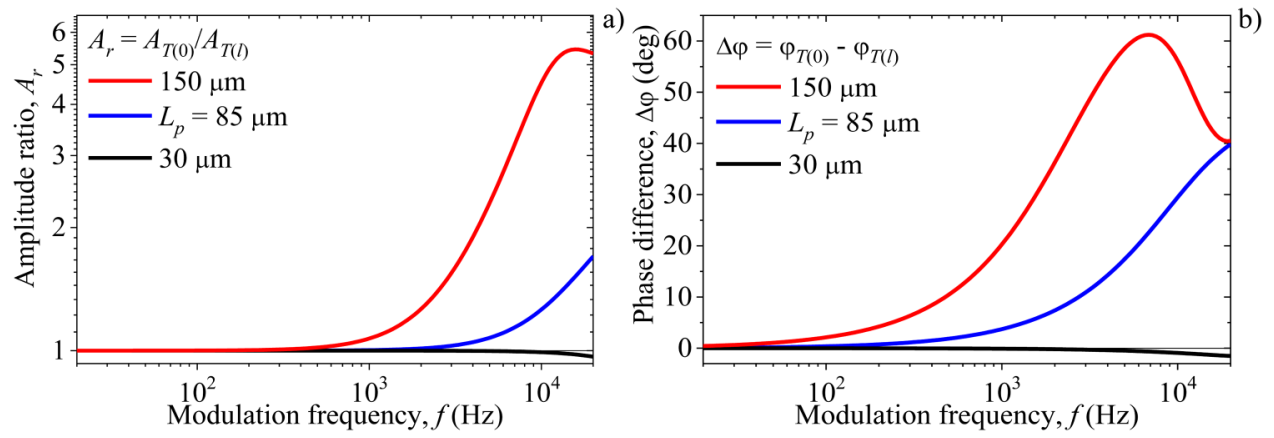

FIG. 4. The results of (a) amplitude ratios $A_{r}$ and (b) phase differences $\Delta \varphi$ between front $T(0)$ and rear $T(I)$ sample surface temperatures in the case of plasma-thick $(150 \mu \mathrm{m})$, plasma-thin $(30 \mu \mathrm{m})$, and carrier diffusion length $\left(L_{p}=85 \mu \mathrm{m}\right)$ sample thicknesses, as a function of modulation frequencies $f$. frequencies where it does not affect $\delta p_{\text {total }}(f)$. This is the reason why we try to find the correlation between excess carrier existence and $\mathrm{PA}$ response changes using the strongly attenuated $\delta p_{\mathrm{TE}}(f)$ component. Knowing that PA responses are generally proportional to the temperature changes in the sample, and temperature changes are strongly influenced by $\delta n_{p}(x)$, temperature distribution $T_{s}(x)$ calculations (Appendix) can be used to confirm mentioned correlation and possible causality. The most efficient way to present the temperature distribution fluctuations due to excess carrier influence is to calculate $T_{s}(0), T_{s}(l)$ and their amplitude ratios $A_{r}=A_{T(0)} / A_{T(l)}$ and phase differences $\Delta \varphi=\varphi_{T(0)}-\varphi_{T(l)}$. The amplitude ratios and phase differences are used here as a part of long-established signal normalization procedures applied to find small fluctuations unable to see on the logarithmic scale. They afford a measure of departure from the "standard" response. In our case, ${ }^{23}$ the "standard" response would imply $A_{r} \geq 1$ and $\Delta \varphi \geq 0\left(T_{s}(0)>T_{s}(l)\right)$.

Using experimentally obtained $\tau, D_{\mathrm{T}}, s_{1}, s_{2}$, and $D_{p}$ and the well-known theoretical model (Appendix) in the whole photoacoustic frequency domain $(20 \mathrm{~Hz}-20 \mathrm{kHz}), A_{r}$ and $\Delta \varphi$ frequency responses for all investigated sample thicknesses are depicted in Fig. 4.

According to Fig. 4, $A_{r} \geq 1, \Delta \varphi \geq 0\left(T_{s}(0)>T_{s}(l)\right)$ for a plasma-thick sample ("standard" response, $l_{1}=150 \mu \mathrm{m}-$ red line, peak-like behavior), while $A_{r} \leq 1, \Delta \varphi \leq 0\left(T_{s}(0)<T_{s}(l)\right)$ for a plasma-thin one ("non-standard" response, $l_{3}=30 \mu \mathrm{m}$-black line). The transition between plasma-thin and plasma-thick samples is given by the blue line obtained for $l_{2}=85 \mu \mathrm{m}=L_{p}$ ("standard" response). The boundary thickness $L_{p}$ can thus be recognized as the smooth curve without a peak-like behavior that follows the growing trend of the plasma-thick regime. These results are consistent with our previous theoretical predictions about the frequency responses of $A_{r}$ and $\Delta \varphi$ as functions of the sample thickness. ${ }^{23}$

Here, we must pay attention to the possible important effects of the multiple reflections of the modulated light beam on the temperature field of the sample. These effects are strongly determined by the product $\beta l$ between the absorption coefficient $(\beta)$ and thickness $(l)$ of the sample $\mathrm{e}^{29-31}$ such that they are significant at the illuminated (non-illuminated) sample surface for $\beta l \leq 2(\beta l \leq 5)$ only. ${ }^{31}$ In our case, the absorption coefficient of our Si samples is $\beta=2.58 \times 10^{5} \mathrm{~m}^{-1}$ at the wavelength of $660 \mathrm{~nm}$ (red) of the used light source. This yields values $\beta l>5$, for the three samples considered in our experiments $\left(\beta l_{1} \approx 39, \beta l_{2} \approx 22\right.$ and $\left.\beta l_{3} \approx 8\right)$, and, therefore, the semi-infinite sample approximation used in the present work is well justified.

To clarify the results shown in Fig. 4, temperature difference .. is more suitable to use. The results of $\Delta T$ for all sample thicknesses are depicted in Fig. 5(a); (1) $\Delta T>0 \Rightarrow T_{s}(0)>T_{s}(l)$ in the case of plasma-thick (red solid line) and $L_{p}$ thickness sample (blue solid line), (2) $\Delta T<0 \Rightarrow T_{s}(0)<T_{s}(l)$ in the case of plasma-thin samples (black solid line) in the whole photoacoustics frequency domain. Note that presented result $(\Delta T<0)$ is visible only under the conditions when the illuminated sample surface is passivated $\left(s_{1}=2 \mathrm{~m} / \mathrm{s}\right)$ and non-illuminated is $\operatorname{not}\left(s_{2}=24 \mathrm{~m} / \mathrm{s}\right)$. Any other combination $\left(s_{1}=s_{2}, s_{1}>s_{2}\right)$ will not yield the $\Delta T<0$ effect. $^{22,23}$ In the frequency range under consideration, the maximal temperature differences are $\Delta T_{150}^{\max }=80 \times 10^{-8} \mathrm{~K}$ for plasmathick samples and $\Delta T_{30}^{\max }=-3.5 \times 10^{-8} \mathrm{~K}$ for plasma-thin ones. The results depicted in Figs. 4 and 5 confirm our assumption that plasma-thin samples at given frequencies can be considered like
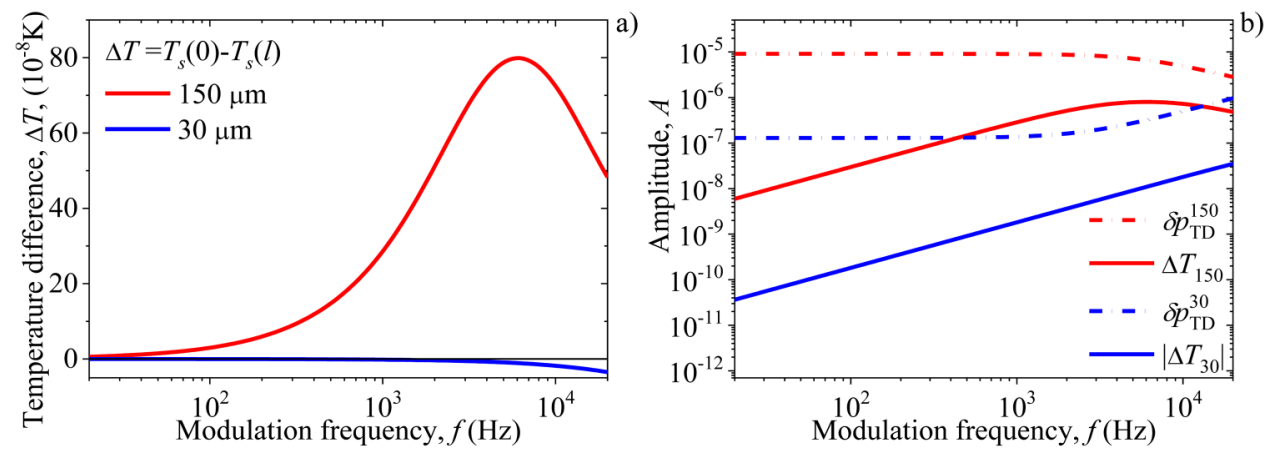

FIG. 5. The results of (a) temperature differences $\Delta T$ and $\Delta T^{*}$ and (b) amplitude behavior of $\delta p_{\text {total }}, \delta p^{*}$ total, $\delta T(x)^{*}$, and $|\Delta T(x)|$ as a function of modulation frequencies $f$. 
efficient heat sinks, using excess carriers as heat carriers to remove the absorbed light energy from the illuminated surface and transfer it to the non-illuminated one, where they release it in the process of recombination. During such transport, $T_{s}(0)$ decreases because the front surface of the sample does not accelerate the recombination, and a large number of carriers manage to escape from it to the sample volume. At the same time, $T_{s}(l)$ increases because carriers that have escaped from the illuminated surface reach the nonilluminated one (thin samples) and recombine there quickly because the surface is non-passivated, i.e., there are a large number of dangling bonds that contribute to a higher recombination efficiency.

The most illustrative depiction of the $\delta p_{\mathrm{TE}}(f)$ component correlation with $\Delta T$ changes is given in Fig. $5(\mathrm{~b})$, where the amplitudes of plasma-thick $\left(\delta p_{\mathrm{TE}}^{150}(f), \Delta T_{150}\right)$ and plasma-thin samples $\left(\delta p_{\mathrm{TE}}^{30}(f),\left|\Delta T_{30}\right|\right)$ are given as a function of the modulation frequency $f$. Knowing that $\Delta T$ changes are a direct consequence of the excess carrier influence, theoretically predicted causality between the excess carrier existence and the thermoelastic component attenuation in the frequency domain is clearly proved.

\section{CONCLUSIONS}

Based on the comparison of experimental and theoretical photoacoustics signals obtained within the modulation frequency range of $20 \mathrm{~Hz}-20 \mathrm{kHz}$, the significant influence of photogenerated excess carriers on the thermal and elastic properties of n-type silicon plates have been uncovered and analyzed. It has been found that the excess carriers, acting as heat carriers, are the cause of the thermoelastic bending of samples. For plasma-thin samples, the effect of the plasmaelastic component on the total photoacoustic signal has been detected for the first time, at high enough modulation frequencies. Furthermore, we have found that the ratio between plasma-thick and plasma-thin amplitudes of the thermoelastic component is around two orders of magnitude greater than its value in the "no carriers" scenario. A maximal temperature difference of $-35 \mathrm{nK}$ has been found between the illuminated and nonilluminated sample surfaces, which show that thin silicon plates with excess carriers could efficiently be used as heat sinks. This latter fact could find potential applications in the electronic industry (component design), environmental chemistry (thin-films analysis), or MEMS (sensor sensitivity). These results are also expected to hold qualitatively for other types of non-degenerated semiconductors (doping below $10^{24} \mathrm{~m}^{3}$ for both $\mathrm{n}$ - or p-type $\mathrm{Si}$ ), ${ }^{32}$ as the excess carriers that act as heat carriers, generate the thermoelastic bending of samples.

\section{ACKNOWLEDGMENTS}

This work was supported by the Ministry of Education, Science and Technological Development of the Republic of Serbia under the Grant Nos. ON171016 and ON172026.

\section{APPENDIX: TEMPERATURE DISTRIBUTION IN SEMICONDUCTOR SAMPLE-“CARRIER” SITUATION}

Figure $6^{22,23}$ depicts a semitransparent semiconductor plate of thickness $l$ and absorption coefficient $\beta$, uniformly illuminated at a

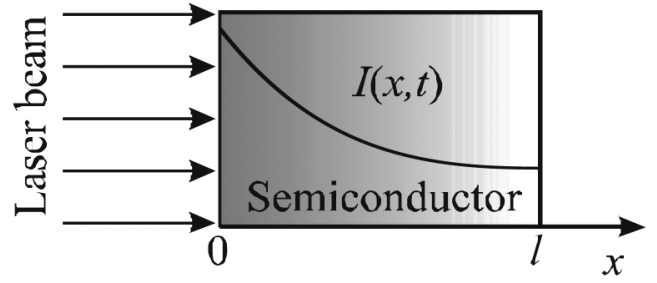

FIG. 6. Scheme of the n-type semiconductor excited with a light source of modulated intensity. 22,2

front surface $x=0$ by a light source of modulated intensity $I$. Inside the sample, according to the Beer-Lambert law, $I$ is given by $I(x, t)=I_{0}[1+\cos (\omega t)] e^{-\beta x}$, where $I_{0}=I_{a}(1-R) / 2, I_{a}$ is the incident light intensity, $R$ is the plate optical reflectivity, $t$ is the time, $\omega=2 \pi f$, and $f$ is the modulation frequency. If the energy of a light source photon $\varepsilon=h v$ is equal to or greater than the bandgap $\varepsilon_{g}$ of the n-type semiconductor $\left(\varepsilon=h v \geq \varepsilon_{g}\right)$, the absorbed photons will generate excess carriers, namely, holes (minority carriers) and electrons (majority carriers) with the same number densities $\delta n_{p}$ and $\delta n_{n}$, respectively.

After they are generated, both types of excess carriers diffuse from regions of a high density to ones of a low density, changing the temperature distribution within the semiconductor and on its surfaces. In the case of n-type semiconductors, carrier diffusion and recombination processes are driven by the minority carrier density given by $^{22,23}$

$$
\delta n_{p}(x)=A_{+} e^{x / L}+A_{-} e^{-x / L}-A e^{-\beta x},
$$

where $\quad A=I_{0} /\left(\varepsilon D_{p} \beta\right), L=L_{p} / \sqrt{(1+i \omega \tau)}, L_{p}=\sqrt{D_{p} \tau}$ is the excess carrier (holes) diffusion length characterized by their diffusion coefficient $D_{p}$ and lifetime $\tau$, and the integration constants $A_{ \pm}$ are defined by ${ }^{22,23}$

$$
A_{ \pm}=\frac{A}{v_{D}} \frac{v_{\beta}\left(v_{D} \pm s_{2}\right) e^{\mp l / L}-v_{D}\left(v_{\beta}-s_{2}\right) e^{-\beta l}}{\left(v_{D}+s_{2}\right) e^{l / L}-\left(v_{D}-s_{2}\right) e^{-l / L}}
$$

depending strongly on the relative values of the characteristic diffusion speeds $v_{D}=D_{p} / L$ and $v_{\beta}=\beta D_{p}\left(v_{\beta}>v_{D}\right)$. In deriving Eq. (A2), only periodic component of the excess carrier density is considered as the quantity of interest in PA and PT similar detection techniques. To simplify the calculation and intensify the excess carrier effects, the sample front surface (Fig. 6, ..) is assumed to be highly passivated $\left(s_{1}=0\right)$, while the back non-illuminated surface $(x=l)$ is assumed to be nonpassivated $\left(s_{2} \gg 0\right)$.

The excess carrier density $\delta n_{p}(x)$ is related to the periodic component of the temperature field $T_{s}(x)$. A standard "thermal source" approach in semiconductors, used in our calculations, implies a $1 \mathrm{D}$ thermal diffusion equation that can be written in the 
form $^{20,22,23}$

$$
\frac{\mathrm{d}^{2} T_{s}(x)}{\mathrm{d} x^{2}}-\sigma^{2} T_{s}(x)=-\frac{\varepsilon-\varepsilon_{g}}{k \varepsilon} \beta I_{0} e^{-\beta x}-\frac{\varepsilon_{g}}{k \tau} \delta n_{p}(x) .
$$

The total solution of Eq. (A3) is given as follows: ${ }^{23}$

$$
T_{s}(x)=T_{\mathrm{term}}(x)+T_{\mathrm{sr}}(x)+T_{\mathrm{br}}(x),
$$

where thermalization, surface, and bulk recombination component are defined with ${ }^{20,23}$

$$
T_{\text {term }}(x)=\frac{I_{0}}{k} \frac{\varepsilon-\varepsilon_{g}}{\varepsilon} \frac{\beta}{\beta^{2}-\sigma^{2}}\left\{b \frac{\cosh [\sigma(x-l)]-e^{-\beta l} \cosh (\sigma x)}{\sinh (\sigma l)}-e^{-\beta x}\right\},
$$

$$
T_{\mathrm{sr}}(x)=\frac{\varepsilon_{g}}{k \sigma} \frac{s_{2} \delta n_{p}(l) \cosh (\sigma x)}{\sinh (\sigma l)},
$$

and

$T_{\mathrm{br}}(x)=\frac{\varepsilon_{g} B_{1}}{\tau k \sigma^{2}}\left[\frac{B_{2} e^{\sigma x}+B_{3} e^{-\sigma x}}{2 \sin h(\sigma l)}-\frac{1}{c^{2}-1}\left(\frac{\delta n_{p}(x)}{B_{1}}+\frac{b^{2}-c^{2}}{b^{2}-1} e^{-\beta x}\right)\right]$.

Here $b=\beta / \sigma, c=(L \sigma)^{-1}, \sigma=(1+i) / \mu$ being the complex heat diffusion coefficient, $\mu=\sqrt{D_{\mathrm{T}} / \pi f}$ the thermal diffusion length of the semiconductor, and $D_{\mathrm{T}}$ the thermal diffusivity, while the integration constants $B_{n}(n=1,2,3)$ are defined as ${ }^{20,23}$

$$
\begin{gathered}
B_{1}=-\frac{\beta I_{0}}{\varepsilon D_{p}\left(\beta^{2}-L^{-2}\right)}, \\
B_{2}=B_{4} e^{-\sigma l}+B_{5}, \\
B_{3}=B_{4} e^{\sigma l}+B_{5},
\end{gathered}
$$

and

$$
\begin{aligned}
& B_{4}=-c \frac{\frac{1}{B_{1}}\left[\delta n_{p}(l)-\delta n_{p}(0) \cosh (l / L)\right]-\cosh (l / L)+e^{-\beta l}}{\sinh (l / L) \cdot\left(c^{2}-1\right)}-\frac{b}{b^{2}-1}, \\
& B_{5}=c \frac{\frac{1}{B_{1}}\left[\delta n_{p}(l) \cosh (l / L)-\delta n_{p}(0)\right]-1+e^{-\beta l} \cosh (l / L)}{\sinh (l / L) \cdot\left(c^{2}-1\right)}+\frac{b e^{-\beta l}}{b^{2}-1} .
\end{aligned}
$$

\section{DATA AVAILABILITY}

The data that support the findings of this study are available from the corresponding author upon reasonable request.

\section{REFERENCES}

${ }^{1}$ S. Bialkowski, Photothermal Spectroscopy Methods for Chemical Analysis (John Wiley, New York, 1996).

${ }^{2}$ S. S. Li, "Excess carrier phenomenon in semiconductors," in Semiconductor Physical Electronics, edited by S. S. Li (Springer, New York, NY, 2006).

${ }^{3}$ D. K. Ferry, Semiconductors, Bonds and Bands (IOP Publishing Ltd, Bristol, 2013).

${ }^{4}$ I. Lashkevych, O. Titov, and Y. G. Gurevich, "Recombination and temperature distribution in semiconductors," Semicond. Sci. Technol. 27, 055014 (2012).

${ }^{\mathbf{5}}$ S. Volz, Microscale and Nanoscale Heat Transfer (Topics in Applied Physics) (Springer, 2010), ISBN: 978-3642071584.

${ }^{6} \mathrm{M}$. Kaviany, Heat Transfer Physics, 2nd ed. (Cambridge University Press, Cambridge, 2014), ISBN: 978-1-107041783.

${ }^{7}$ D. Foumier, A. C. Boccara, A. Skumanish, and N. M. Amer, "Photothermal investigation of transport in semiconductors: Theory and experiment," J. Appl. Phys. 59, 787 (1986).

${ }^{8}$ A. Rosencwaig, "Thermal wave characterization and inspection of semiconductor materials and devices," in Photoacoustic and Thermal Wave Phenomena in Semiconductors, edited by A. Mandelis (Elsevier Publishing Company, 1987), pp. 97-135.

${ }^{9}$ Yu. G. Gurevich and I. Lashkevych, "Sources of fluxes of energy, heat, and diffusion heat in a bipolar semiconductor: Influence of nonequilibrium charge carriers,” Int. J. Thermophys. 34, 341 (2013).

${ }^{10} \mathrm{Yu}$. G. Gurevich and J. E. Velázquez-Pérez, "The role of non-equilibrium charge carriers in thermoelectric cooling," J. Appl. Phys. 114, 033704 (2013).

${ }^{11}$ A. Mandelis, R. Bleiss, and F. Shimura, "Highly resolved separation of carrierand thermal-wave contributions to photothermal signals from Cr-doped silicon using rate-window infrared radiometry," J. Appl. Phys. 74, 3431 (1993).

${ }^{12}$ J. Batista, A. Mandelis, and D. Shaughnessy, "Temperature dependence of carrier mobility in Si wafers measured by infrared photocarrier radiometry," Appl. Phys. Lett. 82, 4077-4079 (2003).

${ }^{13}$ K. M. Gupta, and Nishu Gupta, "Carrier transport in semiconductors," in Advanced Semiconducting Materials and Devices, Part of the Series Engineering Materials (Springer International Publishing, Switzerland, 2016), pp. 87-144.

${ }^{14} \mathrm{M}$. Pawlak, "Photothermal, photocarrier, and photoluminescence phenomena in semiconductors studied using spectrally resolved modulated infrared radiometry: Physics and applications," J. Appl. Phys. 126, 150902 (2019).

${ }^{15}$ L. C. M. Miranda, "Theory of the photoacoustic effect in semiconductors influence of carrier diffusion and recombination,” Appl. Opt. 21, 2923 (1982).

${ }^{16}$ A. Sablikov and V. B. Sandomirskii, "The photoacoustic effect in semiconductors," Phys. Status Solidi B 120, 471 (1983).

${ }^{17}$ E. Marín, H. Vargas, P. Diaz, and I. Riech, "On the photoacoustic characterization of semiconductors: Influence of carrier recombination on the thermodiffusion, thermoelastic and electronic strain signal generation mechanisms," Phys. Status Solidi A 179, 387-402 (2000).

${ }^{18}$ A. Pinto Neto, H. Vargas, N. E. Jxite, and L. C. M. Miranda, "Photoacoustic characterization of semiconductors: Transport properties and thermal diffusivity in GaAs and Si,” Phys. Rev. B 41, 9971 (1990).

${ }^{19}$ D. M. Todorovic, P. M. Nikolic, M. D. Dramicanin, D. G. Vasiljevic, and Z. D. Ristovski, "Photoacoustic frequency heat-transmission technique: Thermal and carrier transport parameters measurements in silicon," J. Appl. Phys. 78, 5750 (1995).

${ }^{20}$ D. M. Todorović, P. M. Nikolić, A. I. Bojičić, and K. T. Radulović, "Thermoelastic and electronic strain contributions to the frequency transmission photoacoustic effect in semiconductors," Phys. Rev. B 55(23), 15631-15642 (1997).

${ }^{21}$ D. M. Todorović and P. M. Nikolić, Semiconductors and Electronic Materials, Progress in Photothermal and Photoacoustic Science and Technology (Optical Engineering Press, New York, 2000), pp. 273-318.

${ }^{22}$ D. K. Markushev, D. D. Markushev, S. Galović, S. Aleksić, D. S. Pantić, and DM Todorović, "The surface recombination velocity and bulk lifetime influences on photogenerated excess carrier density and temperature distributions in n-type 
silicon excited by a frequency-modulated light source," Facta Univ. Ser. Electron. Energ. 31, 313-328 (2018).

${ }^{23}$ D. K. Markushev, D. D. Markushev, S. M. Aleksic, D. S. Pantic, S. Galovic, D. M. Todorovic, and J. Ordonez-Miranda, "Effects of the photogenerated excess carriers on the thermal and elastic properties of n-type silicon excited with a modulated light source: Theoretical analysis," J. Appl. Phys. 126, 185102 (2019).

${ }^{24}$ D. D. Markushev, J. Ordonez-Miranda, M. D. Rabasovic, M. Chirtoc, D. M. Todorovic, S. E. Bialkowski, D. Korte, and M. Franko, "Thermal and elastic characterization of glassy carbon thin films by photoacoustic measurements," Eur. Phys. J. Plus 132, 33 (2017).

${ }^{25}$ M. D. Rabasović, M. G. Nikolić, M. D. Dramićanin, M. Franko, and D. D. Markushev, "Low-cost, portable photoacoustic setup for solid samples," Meas. Sci. Technol. 20, 095902 (2009).

${ }^{26}$ D. D. Markushev, J. Ordonez-Miranda, M. D. Rabasović, S. Galović, D. M. Todorović, and S. E. Bialkowski, "Effect of the absorption coefficient of aluminium plates on their thermoelastic bending in photoacoustic experiments," J. Appl. Phys. 117, 245309 (2015).
${ }^{27}$ D. D. Markushev, M. D. Rabasović, D. M. Todorović, S. Galović, and S. E. Bialkowski, "Photoacoustic signal and noise analysis for Si thin plate: Signal correction in frequency domain," Rev. Sci. Instrum. 86, 035110 (2015).

${ }^{28}$ S. M. Aleksić, D. K. Markushev, D. S. Pantić, M. D. Rabasović, D. D. Markushev, and D. M. Todorović, "Ëlectro-acoustic influence of the measuring system on the photoacoustic signal amplitude and phase in frequency domain," Facta Univ. Ser. Phys. Chem. Technol. 14(1), 9-20 (2016).

${ }^{29}$ D. M. Todorovic, M. D. Rabasovic, D. D. Markushev, and M. Sarajlic, J. Appl. Phys. 116, 053506 (2014).

${ }^{30}$ I. Lashkevych, "The general finding technique of effective parameters for nonuniform solids," Ukr. J. Phys. 51(1), 47-55 (2006). http://archive.ujp.bitp.kiev. ua/index.php?item $=$ j\&id $=75$.

${ }^{31}$ M. A. Zambrano-Arjona, J. Ordonez-Miranda, R. A. Medina-Esquivel, F. Peñuñuri, P. Martinez et al. "Effect of the multiple reflections of a light beam on the thermal wave field of a sample of finite thickness," J. Appl. Phys. 111, 094915 (2012).

${ }^{32}$ S. Wang, Fundamentals of Semiconductor Theory and Device Physics (Prentice-Hall International, Inc., 1989), pp. 265-287, ISBN: 0-13-344425-2. 